\title{
Performance Analysis of Overcoming Far-end Congestion in Large-Scale Networks
}

\author{
Zaheeruddin \\ Ph.D. Scholar \\ Department of E.C.E. \\ AISECT University, Bhopal
}

\author{
Sanjeev Gupta, PhD \\ Prof. Department of E.C.E \\ AISECT University, Bhopal
}

\begin{abstract}
Interconnection networks modify fast data communication between components of a digital system. Today, interconnections networks are utilized during a vary of applications like switch and router materials, processormemory interconnect, I/O interconnects, and on-chip networks, to call a couple of. The design of an interconnection network has three aspects - the topology, the routing rule used, and additionally the flow management mechanism used. Though, earlier work doesn't significance the impact of farend congestion or the congestion beginning the high channel latency between the routers. Due to the long inter-router latency, the in-flight packets (and credits) result in inaccurate congestion data and might cause inaccurate adaptive routing selections. We tend to propose a history window based approach to remove the impact of phantom congestion. We've a trend to mutually show but using the standard of native queue occupancies and adding together an offset extensively eradicate the impact of transient congestion.
\end{abstract}

\section{Keywords}

Interconnect, topology, Adaptive Routing

\section{INTRODUCTION}

Wireless technologies have revolutionized the planet of communications. It started with the use of radio receivers or transceivers to be used in wireless telegraphy early on; and currently the term wireless is used to explain technologies like the cellular networks and wireless broadband internet. Although the wireless medium has limited spectrum along with many different constraints as compared to the guided media, it provides the sole suggests that of mobile communication. Wireless ad hoc networking is used for random and fast deployment of an oversized range of nodes, that may be a technology with a wide vary of applications like tactical communications, disaster relief operations, health care and temporary networking in areas that aren't densely inhabited. A mobile ad-hoc network (MANET) consists of mobile hosts equipped with wireless communication devices. The communication of a mobile host is received by all hosts among its transmission variety because of the broadcast nature of wireless communication and Omni-directional antennae. If 2 wireless hosts aren't among the transmission range in ad hoc networks, different mobile hosts set between them will forward their messages that effectively build connected networks among the mobile hosts within the deployed area.

The use of wireless ad hoc networks additionally introduces further security challenges that ought to be dealt with.

The advancement in wireless communications and electronics has enabled the event of low-priced, low-power and multifunctional sensors. Basically, every sensor node contains sensing, processing, transmission, power unit, and a few optional elements (e.g. mobilizer, position system). variety of those sensors are often networked to full some unattended operations for specific applications, hence forming WSNs. WSNs support a range of information collection applications, and have profound effects on each military and civil applications, like environmental observance, tactical military observance, traffic police work, video surveillance and physical security.

Typically, WSNs contain a large range of sensor nodes. These sensors have the power to communicate with one another and can also be connected to gateways (sinks or base stations) of WSNs. The measurement and observation data packets from multiple sensors are then processed and forwarded to external networks via sinks that act as gateways. This procedure is called data gathering.

Although congestion could be a common drawback all told varieties of networks, up to currently it's not been a crucial issue in interconnection network style. The explanations for this are multiple. Firstly, the dimensions of most systems (real or modeled) ranged from 64 to 512 nodes, all having single injection queues, whose HOLB (head-of-line blocking) was providing enough hidden management to stop the node from flooding the network. Secondly, the software system overheads were high, so it absolutely was rare for a true system to supply loads near the $100 \%$ utilization. Thirdly, networks with few resources ( 2 or three virtual channels and buffers of a couple of phits) unbroken congestion low as a result of blocked messages unfold on their methods, limiting network output because of contention. Thus, it's not shocking that almost all of the effort went into reducing contention by increasing adaptivity and handling the issues this brought in, like deadlock.

Interconnection networks are the communication infrastructure of any digital system and have a big impact on the system performance and price [1]. The interconnection network could be a key component of a tightly coupled digital computer system. It provides low latency and high bandwidth communication for a range of workloads. Because the normal microchip is being replaced by multithreaded ones or by chip multiprocessors, each the amount of injectors 1 and therefore the total offered load per node has increased considerably. Interconnection networks are an important component of latest pc systems. From giant scale systems to multicore architectures [16], the interconnection network that connects processors and memory modules significantly impacts the final performance and price of the system. As processor and memory performance continues to increase, multicomputer interconnection networks are becoming even a lot of crucial as they principally verify the bandwidth and latency of remote access. a decent interconnection network is intended around the capabilities and constraints of accessible technology. Increasing material router pin bandwidth, for example, has 
motivated the utilization of high-radix routers [15] during which the increased bandwidth is used to extend the amount Of ports per router, instead of maintaining a little range of ports and increasing the bandwidth per port. The Cray black widow system, one in all the primary systems to use a highradix network, uses a variant of the folded-Clos topology and radix-64 routers a big departure from previous low-radix 3D torus networks. Recently, the appearance of economical optical signaling permits topologies with long channels.

\section{THEORY}

A node at some point in a wireless sensor network (WSN) might be a small embedded computing device that interfaces with sensors/actuators and communicates using short-range wireless transmitters. Such nodes act alone conversely helpfully to create a logical network during which information packets are routed hop-by-hop towards management nodes, generally known as sinks or base stations. A WSN contains a probably large set of nodes that will be distributed over a good geographic area, indoor or outdoor. Wireless sensing element networks modify varied sensing and observance services in areas of great importance like economical business production, safety and security reception, and in traffic and environmental observance. Traffic patterns in sensing element networks are typically derived from the physical processes that they sense. Sensing element networks typically operate under light load and suddenly become active in response to a detected or monitored event. Looking on the application, this could lead to the generation of large, sudden, and related to impulses of information that has to be delivered to a small number of sinks while not significantly disrupting the performance (i.e., fidelity of the sensing application. This high generation rate of information packets is usually uncontrolled and sometimes ends up in congestion. During this state, collisions occur within the medium or in case of existence of an efficient mac protocol, the node buffers overflow [22, 27], leading to random drops of information packets and increased delay. Dropped packets are a serious handicap for these networks since they lead to severe energy consumption. Within the case that no countermeasures are taken, the power of congested nodes is often exhausted leading to the creation of routing "holes" within the network.

Congestion within the background of networks refers to a network position wherever a node carries lots information that it will make the network service quality increasingly worse. It leads to queuing delay and loss of information packets. It's going to additionally cause overcrowding of latest connections.

Congestion happens once the amount of packets being transmitted from side to side the network approaches the packet handling ability of the network. Within the state of affairs of congestion we tend to get the decrease in throughput.

\section{METHOD}

\subsection{MIN Congestion Aware Algorithm}

MIN Congestion aware algorithm takes into consideration even the traffic stuck up at the router ports unlike the previous 2 approaches. It also maintains a history window which keeps track of the queues of the nodes. The maintenance should be taken care by a device which has the capability to recharge itself or to be replaced easily and should have highest life. In fact the maintenance has to be done by router.

The MIN Congestion Aware algorithm can be described as below:-
1) Source Node, Destination Node and Transmission Range acts as input parameters.

2) The neighbor nodes are found out for the given source node.

3) If neighbor nodes have destination stop. Otherwise proceed to step4.

4) Find out the node which has the lowest MIN Criteria.

5) Check whether the destination node belongs to same router or a different one. If belongs to same then continue with step1.

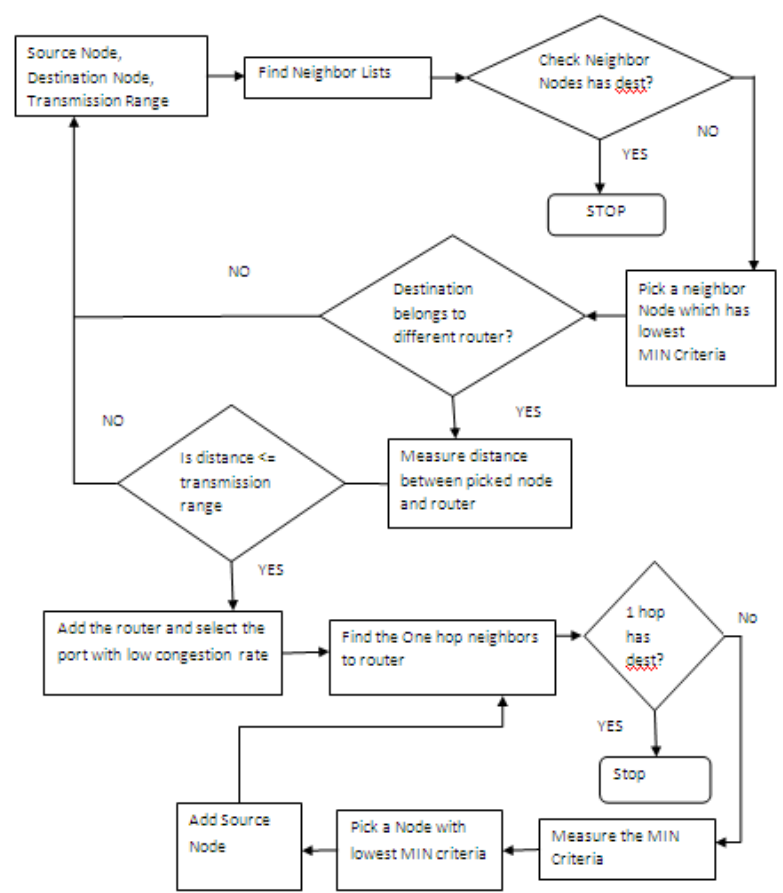

Fig.1 MIN congestion aware Routing algorithm

6) If they belong to different router then measure the distance between the picked node and router.

7) Check the distance is less than or equal to transmission range add the router and establish a connection with a port which has lowest queue.

8) Find the one hop neighbors of the router.

9) If the one hop neighbors have destination then stop the process. Otherwise proceed to step10.

10) Obtain the MIN criteria for each of the neighbors.

11) Pick a node which has the lowest MIN criteria.

12) The new node acts like a source node. Go back to Step8.

13) The MIN Criteria for minimum congestion is defined as follows:

$$
\begin{aligned}
& \text { MIN }_{\text {criteria }}=q_{\text {length }} H_{\text {length }}+T_{\text {packetmove }} \\
& \text { Where, } \\
& q_{\text {length }}=\text { length of the queue } \\
& H_{\text {length }}=\text { number of hops w.r.t router or current source } \\
& T_{\text {packetmove }}=\text { Time for removing packets from queue to an outgoing link }
\end{aligned}
$$

14) The MIN criteria for router port selection is defined as 
below:

$M I N_{\text {routerport }}=\frac{q_{\text {length }} H_{\text {length }}}{q_{\text {avg }}}$

Where,

$q_{\text {lavg }}=$ Average queue length across all queue sof the router

$H_{\text {length }}=$ number of hops w.r.t router or current source

$q_{\text {length }}=$ queue length of a specific node

\section{RESULT}

Result is evaluated by comparing the performance of MIN, VAL and PAR routing on different network parameters such as route discovery time, number of hops, number of alive nodes, number of dead nodes, energy consumed by the nodes and routing overhead. All the parameters are compared for three routing algorithms namely PAR, VAL and MIN.



Fig. 2 Comparison between MIN, PAR and VAL on route discovery time in $\mathrm{ms}$

Above graph shows comparison of MIN, VAL and PAR on the basis of route discovery time in ms. Above shown graph is plotted between number of iterations and route discovery time. Number of iterations is shown in horizontal direction and route discovery time is shown in vertical direction. Blue line indicates performance of MIN, orange line indicates performance of PAR, and pink line indicates performance of VAL.



Fig.3 Comparison between MIN, PAR and VAL on number of hops

Above graph shows comparison of MIN, VAL and PAR on the basis of number of hops. Above shown graph is plotted between number of iterations and number of hops. Number of iterations is shown in horizontal direction and route discovery time is shown in vertical direction. Blue line indicates performance of MIN, orange line indicates performance of $\mathrm{PAR}$, and pink line indicates performance of VAL.



Fig.4 Comparison between MIN, PAR and VAL on energy consumed in $\mathbf{~ m j}$

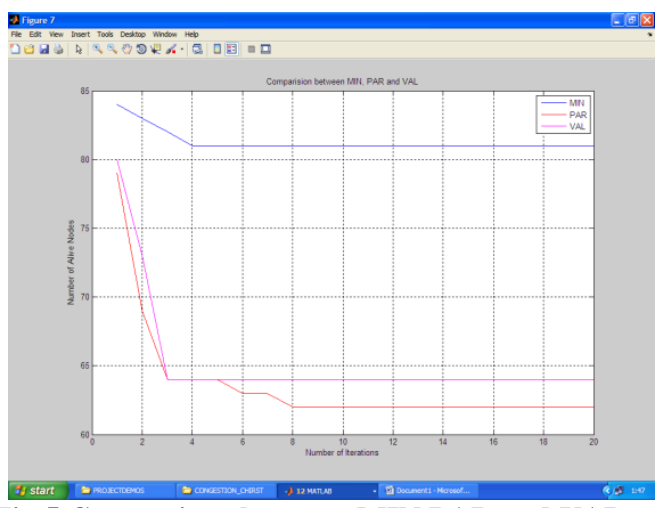

Fig.5 Comparison between MIN,PAR and VAL on number of alive nodes

Above graph shows comparison of MIN, VAL and PAR on the basis of number of alive nodes. Above shown graph is plotted between number of iterations and number of alive nodes. Number of iterations is shown in horizontal direction and number of alive nodes is shown in vertical direction. Blue line indicates performance of MIN, orange line indicates performance of PAR, and pink line indicates performance of VAL.

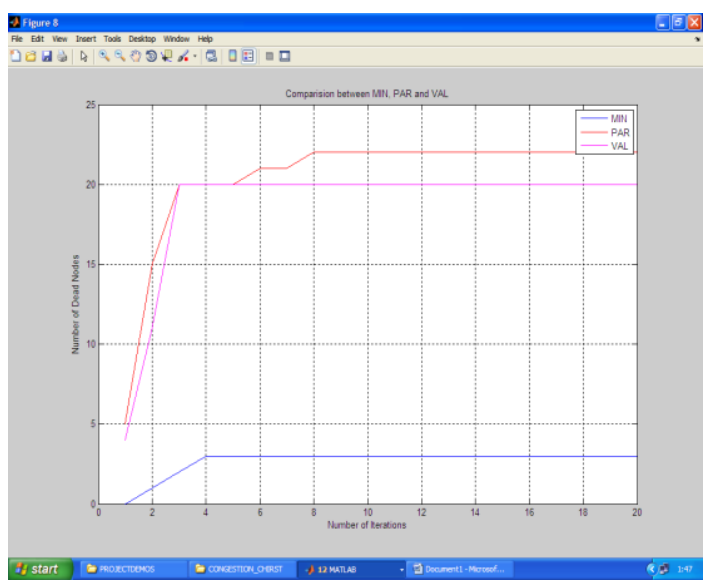

Fig.6 Comparison between MIN,PAR and VAL on number of dead nodes 
Above graph shows comparison of MIN, VAL and PAR on the basis of number of dead nodes. Above shown graph is plotted between number of iterations and number of dead nodes. Number of iterations is shown in horizontal direction and number of dead nodes is shown in vertical direction. Blue line indicates performance of MIN, orange line indicates performance of PAR, and pink line indicates performance of VAL

\section{CONCLUSION}

In this article result examination of Overcoming Far-end Congestion in Large-Scale Networks which present above results, which demonstrate the difficulty of the earlier ways ought to be increased. We tend to determine the impact of farend congestion that occurs in large-scale networks due to long latency between neighboring routers and also the totally different length channels within the topology. Our results show that the planned techniques considerably improve the latency and output of the PAR adaptive routing on the dragonfly network. Our results are comparing the performance of MIN, VAL and PAR routing on different network parameters.

\section{REFERENCES}

[1] Jongmin Won, Gwangsun Kim, John Kim, S. K., anTed Jiang, Mike Parker, Steve Scott. "Overcoming Far-end Congestion in Large-Scale Networks" IEEE 2015

[2] Camarero, Cristóbal, Enrique Vallejo, and Ramón Beivide. "Topological characterization of hamming and dragonfly networks and its implications on routing" ACM Trans. on Arch. and Code Optimization (TACO) 11, no. 4 (2015): 39

[3] F. Pablo, E. Vallejo, C. Camarero, R. Beivide, and M. Valero. "Throughput unfairness in dragonfly networks under realistic traffic patterns" In Cluster Computing (CLUSTER), 2015 IEEE Inte. Conf. on, pp. 801-808. IEEE, 2015.

[4] G. Marina, E. Vallejo, R. Beivide, M. Odriozola, and M. Valero. "Efficient routing mechanisms for dragonfly networks" In Inter. Conf. on Parallel Processing (ICPP), 2013 42nd on, pp. 582-592. IEEE, 2013.

[5] Dorier, Matthieu, Misbah Mubarak, Rob Ross, Jianping Kelvin Li, Christopher D. Carothers, and Kwa-Liu Ma. "Evaluation of Topology-Aware Broadcast Algorithms for Dragonfly Networks" In Cluster Computing (CLUSTER), 2016 IEEE Inter. Conf. on, pp. 40-49. IEEE, 2016.

[6] Prisacari, Bogdan, German Rodriguez, Cyriel Minkenberg, Marina Garcia, Enrique Vallejo, and Ramon Beivide. "Performance optimization of load imbalanced workloads in large scale Dragonfly systems" In High Performance Switching and Routing (HPSR), 2015 IEEE 16th Inter. Conf. on, pp. 1-6. IEEE, 2015.

[7] Xiang, Dong, and Xiaowei Liu. "Deadlock-free broadcast routing in dragonfly networks without virtual channels" IEEE Trans. on Parallel and Distributed Syst. 27, no. 9 (2016): 2520-2532.

[8] Hastings, Emily, David Rincon-Cruz, Marc Spehlmann, Sofia Meyers, Anda Xu, David P. Bunde, and Vitus J. Leung. "Comparing global link arrangements for Dragonfly networks" In Cluster Computing (CLUSTER), 2015 IEEE Inter. Conf. on, pp. 361-370. IEEE, 2015.

[9] Faanes, Greg, Abdulla Bataineh, Duncan Roweth, Edwin Froese, Bob Alverson, Tim Johnson, Joe Kopnick, Mike Higgins, and James Reinhard. "Cray cascade: a scalable HPC system based on a Dragonfly network" p. 103. IEEE Computer Society Press, 2012.

[10] Bhatele, Abhinav, Nikhil Jain, Yarden Livnat, Valerio Pascucci, and Peer-Timo Bremer. "Analyzing network health and congestion in dragonfly-based supercomputers" IEEE International, pp. 93-102. IEEE, 2016.

[11] B. Arimilli, Ravi Arimilli, Vicente Chung Scott Clark, Wolfgang Denzel, Ben Drerup , Torsten Hoef "The percs high-performance interconnect" in High Performance Interconnects (HOTI), 2010 IEEE 18th Annual Symposium on, Mountain View, CA, August 2010, pp. $75-82$.

[12] S. Scott et al., "The black widow high-radix clos network" in Proceedings of ISCA'06, Boston, MA, June 2006, pp. 16-28.

[13] P. Gratz et al., "Regional congestion awareness for load balance in networks-on-chip" in Proceedings of HPCA’08, Salt Lake City, UT, Feb 2008, pp. 203-214.

[14] N. Jiang et al., "Indirect adaptive routing on large scale interconnection networks" in Proceedings of ISCA'09, Austin, TX, June 2009, pp. 220-231.

[15] W. Dally, "Virtual-channel flow control" Parallel and Distributed Systems, IEEE Trans. on, vol. 3, no. 2, pp. 194-205, 1992

[16] D. Helbing, "Traffic and related self-driven manyparticle systems" Rev. Mod. Phys., vol. 73, pp. 10671141, Dec 2001.

[17] J. Bell et al., "Boxlib users guide" 2013, Center for Computational Sciences and Engineering, Lawrence Berkeley National Laboratory.

[18] H. Dong et al., "Quasi diffusion accelerated monte carlo" 2011, Los Alamos National Laboratory.

[19] D. F. Richards et al., "Beyond homogeneous decomposition: Scaling long-range forces on massively parallel systems" in Proceedings of SC'09, Portland, Oregon, November 2009, Article 60.

[20] P. F. Fischer et al., 2008, http://nek5000.mcs.anl.gov. H Adalsteinsson et al., "A simulator for large-scale parallel computer architectures" Int. J. Distrib. Syst. Technol., vol. 1, no. 2, pp. 57-73, Apr. 2010. 